\title{
The first coronal mass ejection observed in both visible-light and UV H I Ly- $\alpha$ channels of the Metis coronagraph on board Solar Orbiter $^{\star}$
}

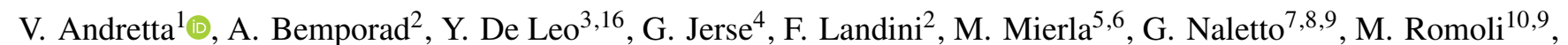
C. Sasso ${ }^{1}$, A. Slemer ${ }^{8}$, D. Spadaro ${ }^{11}$, R. Susino ${ }^{2}$, D.-C. Talpeanu ${ }^{12,5}$, D. Telloni ${ }^{2}$, L. Teriaca ${ }^{3}$, M. Uslenghi ${ }^{13}$, E. Antonucci ${ }^{9}$, F. Auchère ${ }^{14}$, D. Berghmans ${ }^{5}$, A. Berlicki ${ }^{15}$, G. Capobianco ${ }^{2}$, G. E. Capuano ${ }^{11,16}$, C. Casini ${ }^{8,17}$, M. Casti ${ }^{18,9}$, P. Chioetto ${ }^{8,17}$, V. Da Deppo ${ }^{8,9}$, M. Fabi ${ }^{19,20}$, S. Fineschi ${ }^{2}$, F. Frassati ${ }^{2}$, F. Frassetto ${ }^{8,9}$, S. Giordano ${ }^{2}$, C. Grimani ${ }^{19,20}$, P. Heinzel ${ }^{21}$, A. Liberatore ${ }^{2,22}$, E. Magli ${ }^{23}$, G. Massone ${ }^{2}$, M. Messerotti ${ }^{4}$, D. Moses ${ }^{24}$, G. Nicolini ${ }^{2}$, M. Pancrazzi ${ }^{2}$, M.-G. Pelizzo ${ }^{25}$, P. Romano ${ }^{11}$, U. Schühle ${ }^{3}$, M. Stangalini ${ }^{26}$, Th. Straus ${ }^{1}$, C. A. Volpicelli ${ }^{2}$, L. Zangrilli ${ }^{2}$, P. Zuppella ${ }^{8,9}$, L. Abbo ${ }^{2}$, R. Aznar Cuadrado ${ }^{3}$, R. Bruno ${ }^{27}$, A. Ciaravella ${ }^{28}$, R. D'Amicis ${ }^{27}$, P. Lamy ${ }^{29}$, A. Lanzafame ${ }^{16,9}$, A. M. Malvezzi ${ }^{30}$, P. Nicolosi ${ }^{31,8}$, G. Nisticò ${ }^{32}$, H. Peter ${ }^{3}$, C. Plainaki ${ }^{26,9}$, L. Poletto ${ }^{8}$, F. Reale ${ }^{33,9}$, S. K. Solanki ${ }^{3}$, L. Strachan ${ }^{34}$, G. Tondello ${ }^{31}$, K. Tsinganos ${ }^{35}$, M. Velli ${ }^{36}$, R. Ventura ${ }^{11}$, J.-C. Vial ${ }^{14}$,

J. Woch ${ }^{3}$, and G. Zimbardo ${ }^{32,9}$

(Affiliations can be found after the references)

Received 11 October 2021 / Accepted 28 October 2021

\section{ABSTRACT}

Context. The Metis coronagraph on board Solar Orbiter offers a new view of coronal mass ejections (CMEs), observing them for the first time with simultaneous images acquired with a broad-band filter in the visible-light interval and with a narrow-band filter around the $\mathrm{H}$ I Ly- $\alpha$ line at $121.567 \mathrm{~nm}$, the so-called Metis UV channel.

Aims. We show the first Metis observations of a CME, obtained on 16 and 17 January 2021. The event was also observed by the EUI/FSI imager on board Solar Orbiter, as well as by other space-based coronagraphs, such as STEREO-A/COR2 and SOHO/LASCO/C2, whose images are combined here with Metis data.

Methods. Different images are analysed here to reconstruct the 3D orientation of the expanding CME flux rope using the graduated cylindrical shell model. This also allows us to identify the possible location of the source region. Measurements of the CME kinematics allow us to quantify the expected Doppler dimming in the Ly- $\alpha$ channel.

Results. Observations show that most CME features seen in the visible-light images are also seen in the Ly- $\alpha$ images, although some features in the latter channel appear more structured than their visible-light counterparts. We estimated the expansion velocity of this event to be below $140 \mathrm{~km} \mathrm{~s}^{-1}$. Hence, these observations can be understood by assuming that Doppler dimming effects do not strongly reduce the Ly- $\alpha$ emission from the CME. These velocities are comparable with or smaller than the radial velocities inferred from the same data in a similar coronal structure on the east side of the Sun.

Conclusions. The first observations by Metis of a CME demonstrate the capability of the instrument to provide valuable and novel information on the structure and dynamics of these coronal events. Considering also its diagnostics capabilities regarding the conditions of the ambient corona, Metis promises to significantly advance our knowledge of such phenomena.

Key words. Sun: atmosphere - Sun: corona - Sun: UV radiation - Sun: coronal mass ejections (CMEs)

\section{Introduction}

Space-based coronagraphs are the main tools currently available for continuously monitoring the solar corona. Images acquired by these instruments provide unique input for the release of the first alert and forecasting on the occurrence of prominence eruptions and coronal mass ejections (CMEs) and their possible impact on the Earth's magnetosphere (see review by Webb \& Howard 2012). Over the last few decades, space-based coronagraphs have allowed us to continuously monitor CMEs and study their early evolution from $\sim 1.5$ to $\sim 30 R_{\odot}$ over more

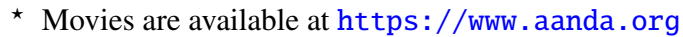

than two solar cycles (Yashiro et al. 2004; Gopalswamy et al. 2009; Lamy et al. 2019).

The success of the Large Angle and Spectrometric Coronagraph Experiment (LASCO) visible-light (VL) coronagraphs (Brueckner et al. 1995) on board the Solar and Heliospheric Observatory (SOHO) mission has demonstrated that these are the only existing instruments capable of providing real-time information on the properties of solar eruptions propagating towards Earth (observed as 'halo CMEs'; see e.g. Schwenn et al. 2005), such as the ejected mass, projected and un-projected speeds and accelerations, propagation direction, and other parameters (Webb et al. 2000; Xie et al. 2004; Michalek et al. 2006). Over the last decade, this information has been complemented with the coronagraphs COR1 and COR2 and the 
heliospheric imagers $\mathrm{HI} 1$ and HI2, providing new information thanks to the unique vantage point offered by the twin Solar Terrestrial Relations Observatory (STEREO) spacecraft (Howard et al. 2008).

In part due to the great success of space-based coronagraphs, most instruments flown so far have been designed to provide images only in polarised and unpolarised VL emission from coronal plasmas. Because this emission is mostly due to the Thomson scattering of photospheric radiation by coronal electrons (the so-called K-corona emission), the information available so far from imaging coronagraphic observations mainly concerns the evolution of the plasma electron density and kinematic properties (e.g., Vourlidas et al. 2000, 2010; Zhang et al. 2004), missing, for instance, information about plasma temperatures and abundances. On the other hand, UV spectral line emission from CMEs has been studied in great detail for many events thanks to the UV Coronagraph Spectrometer (UVCS) instrument (Kohl et al. 1995) on board SOHO. This has allowed us to also study plasma temperatures and elemental composition variations during CMEs, although the instrument field-of-view (FOV) was limited to the spectrometer entrance slit. Despite this limitation, a significant amount of new discoveries were made regarding CMEs thanks to those data. A comprehensive review of CME observations with UVCS can be found in Kohl et al. (2006), and a catalogue is provided by Giordano et al. (2013).

With the 10 February 2020 launch on board Solar Orbiter (Müller et al. 2020) of Metis (Antonucci et al. 2020), a multichannel imaging coronagraph capable of simultaneously observing in the UV in addition to the classical VL bands, it is now possible to obtain a global picture of the corona in both bands. Metis simultaneously provides images of the solar corona in polarised VL broadband in the interval 580-640 $\mathrm{nm}$ and in the UV narrow band centred around the $121.6 \mathrm{~nm}$ Ly- $\alpha$ line emitted by neutral $\mathrm{H}$ atoms (the most intense line in the UV solar spectrum). The instrument square FOV is $\pm 2.9^{\circ}$ wide, with an inner circular occulted area of radius $1.6^{\circ}$; in the course of its eccentric orbit around the Sun, Metis will therefore cover projected altitude intervals going from $1.7-3.0 R_{\odot}$ (at 0.28 au, minimum perihelion) to $2.8-5.5 R_{\odot}$ (at $0.50 \mathrm{au}$ ), with a plate scale of $10.7^{\prime \prime}$ pixel $^{-1}$ and $20^{\prime \prime}$ pixel $^{-1}$ in the VL and UV channels, respectively (Antonucci et al. 2020; Fineschi et al. 2020) ${ }^{1}$.

\section{Description of the event and observations}

On 16-17 January 2021, a slow and relatively faint CME was observed with different space-based instruments and from different locations around the Sun, including the Metis coronagraph on board Solar Orbiter. The event is shown in Fig. 1 as observed by the LASCO/C2 coronagraph on board SOHO (top row) and the COR2 coronagraph on board STEREO-A (bottom row). In the sequence of running-difference VL images, the CME quite clearly appears as an almost circular feature propagating above the east limb as seen by the two instruments in a direction close to the ecliptic plane. This suggests that the observed feature could be interpreted as the signature of a flux rope propagating with its axis almost parallel to the ecliptic plane (Cremades \& Bothmer 2004), a conclusion further supported by the geometrical reconstruction with the graduated cylindrical

\footnotetext{
1 Further analysis of commissioning data has subsequently led to these values being revised to $10.138^{\prime \prime} \pm 0.004$ per pixel in the VL and $20.4^{\prime \prime} \pm$ 0.06 per pixel in the UV channel.
}

shell (GCS) empirical model (Thernisien et al. 2006; Thernisien 2011), as discussed in more detail below.

The position of the Solar Orbiter spacecraft in the ecliptic plane is shown in the top-left panel of Fig. 2, along with the position of STEREO-A and Parker Solar Probe (PSP; Fox et al. 2016), in the geocentric solar ecliptic (GSE) coordinate system (defined such that $X$ is the Earth-Sun line, and $Z$ is aligned with the ecliptic north of date). In that figure, SOHO is close to Earth, at the Lagrangian point L1. The estimated direction of propagation of the CME is also shown.

\subsection{Metis observations}

Between 14 and 17 January 2021, the Metis instrument acquired both VL and UV images at a distance of about $0.6 \mathrm{au}$ from the Sun while performing a synoptic programme. This was specifically designed to obtain out-of-remote-sensing-window observations along the spacecraft orbit in order to monitor the status of the solar corona for a longer duration and provide additional context for the joint science with the in situ instruments on board Solar Orbiter (see also Auchère et al. 2020). Furthermore, the synoptic programme was also intended to provide support for the seventh perihelion of PSP, which occurred on 17 January 2021. An analysis of these data in the context of that event is reported by Telloni et al. (2021).

During the synoptic programme, one VL polarisedbrightness (pB) sequence and two UV images were acquired simultaneously every 4 hours, starting at 00:30 UT on 14 January. The $\mathrm{pB}$ sequence consisted of four polarimetric images with a detector integration time of $30 \mathrm{~s}$, whereas the two UV images were acquired consecutively with a detector integration time of $60 \mathrm{~s}$. To improve image statistics, all images were obtained by averaging 15 on-board frames, resulting in total effective exposure times of 7.5 minutes for each VL image in a $\mathrm{pB}$ sequence and 15 minutes for each UV image in a pair. In both cases, the total duration of the acquisition session was $\sim 30$ minutes. In addition, both detectors were configured with a $2 \times 2$ pixel binning, corresponding to a spatial scale on the plane of the sky (at 0.6 au from the Sun) of $\sim 8700 \mathrm{~km} \mathrm{pixel}^{-1}$ for the VL channel and $\sim 18000 \mathrm{~km} \mathrm{pixel}^{-1}$ for the UV channel. At that distance, the Metis FOV ranges from $\sim 3.4 R_{\odot}$ (internal occulter edge) up to $\sim 7.4 R_{\odot}$ (detector corners); along the ecliptic plane, the outer edge of the FOV was at $\sim 6.1 R_{\odot}$. The acquired data were processed and calibrated following the procedure described in Romoli et al. (2021) with some improvements, as described in Appendix A.

\subsection{The source region}

The event was captured in SOHO/LASCO/C2 images by the automated Computer-Aided CME Tracking (CACTUS) catalogue (Robbrecht \& Berghmans 2004), which provided ${ }^{2}$ a propagation speed of $(100 \pm 20) \mathrm{km} \mathrm{s}^{-1}$, a main propagation latitude of $\sim 10^{\circ} \mathrm{S}$, and a starting time around 23:54 UT on 16 January. This very slow event was not associated with any flare.

In order to determine the location of the possible source region (SR) of the CME, a more refined $3 \mathrm{D}$ reconstruction of the $\mathrm{CME}$ propagation direction is required. This was done here by applying the GCS 3D reconstruction, using the freely distributed data-analysis package, to images acquired by STEREO-A/COR2 and $\mathrm{SOHO} / \mathrm{LASCO} / \mathrm{C} 2$, together with the Metis $\mathrm{pB}$ and $\mathrm{UV}$

\footnotetext{
2 See http://sidc.oma.be/cactus/catalog/LASC0/2_5_0/ qkl/2021/01/CMEO012/CME.html
} 


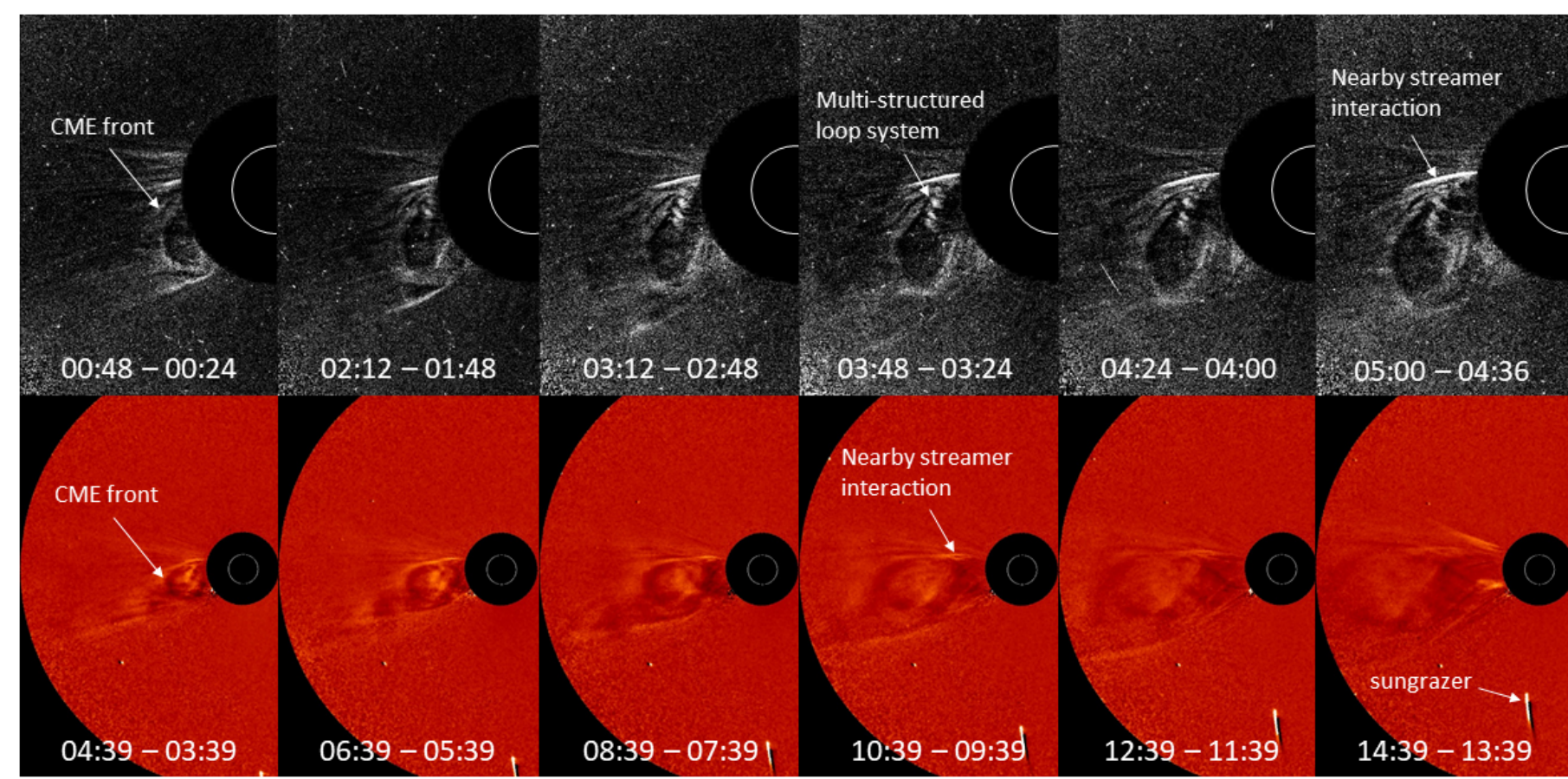

Fig. 1. 16-17 January $2021 \mathrm{CME}$ as observed by the SOHO/LASCO/C2 (top row) and STEREO-A/COR2 coronagraphs (bottom row), with running-difference images taken on 17 January. The angular separation between the two spacecraft at that epoch was about $56^{\circ}$ (see also Fig. 2). The LASCO/C2 difference frames (FOV: $2-6 R_{\odot}$ ) have been obtained via subtraction between the two frames acquired at the times given at the bottom of each panel. The COR2 difference frames (FOV: $2-15 R_{\odot}$ ) are distributed by the official STEREO mission website. The sequence shows first the propagation in the LASCO/C2 FOV (top) followed by the subsequent propagation in the COR2 FOV (bottom).

Ly- $\alpha$ images. Full-disk images obtained with the Extreme Ultraviolet Imager (EUVI) on board STEREO-A at $19.5 \mathrm{~nm}$ and $30.4 \mathrm{~nm}$ were also used for context. In order to enhance the visibility of fainter structures, we used the running-difference images obtained from the three instruments as input to the $3 \mathrm{D}$ reconstruction tool. Considering the different acquisition times of all the instruments, the best, almost co-temporal coverage of the event was provided by the COR2, LASCO/C2, and Metis images acquired at 04:46 UT, 04:48 UT, and 04:45 UT, respectively.

Results obtained from the GCS reconstruction are shown in Fig. 2. With a single set of model parameters it is possible to obtain a satisfactory agreement between the flux-rope model and all the coronagraphic observations acquired from different view points. The flux rope best fitting the VL features of the CME turns out to have originated from a SR located at a heliographic latitude of $13.4^{\circ} \mathrm{S}$ and Carrington longitude of $292.5^{\circ}$ (corresponding to a Stonyhurst heliographic longitude of $134.2^{\circ} \mathrm{E}$ ). The geometry of the flux rope is defined by the following GCS model parameters: an angular width between the flux-rope legs of $2 \alpha=31.4^{\circ}$, a height of the CME front of $h=6.3 R_{\odot}$, a tilt angle relative to the solar equator of $\gamma=-38.6^{\circ}$, and an aspect ratio of $\kappa=0.2$.

The SR thus determined is almost consistent with the disk location of active region (AR) NOAA 12797 (see the red asterisk in the top-left panel of Fig. 2), but the two do not overlap, the former being $\sim 30^{\circ}$ eastwards with respect to the latter. The discrepancy could be justified by considering that CMEs are often deviated and/or rotated with respect to the location of their SR and the orientation of the neutral line in response to their interaction with the large-scale configuration of the corona (e.g. Cremades et al. 2006). However, closer inspection of EUVI $30.4 \mathrm{~nm}$ images (see Fig. 3) reveals that the SR falls almost exactly in a filament channel east of the AR. More evidence supporting the association of the filament with the CME SR comes from another Solar Orbiter instrument: the Extreme Ultraviolet Imager (EUI; Rochus et al. 2020).

On 17 January, EUI was observing with its Full Sun Imager (FSI) in the $17.4 \mathrm{~nm}$ bandpass at 15 min cadence. Most images ${ }^{3}$ were acquired with very high compression ratios (374.5), achieved by a combination of an effective compression scheme, $2 \times 2$ re-binning, and recoding (Nicula et al. 2005). Figure 4 shows both the SR as imaged by EUI before the launch of the event and the CME at a later time as imaged by the Metis UV channel. The sequence of images in the top panels shows a faint jet-like feature gradually rising and then, approximately around the estimated CME starting time (last two panels), deviating north, as if following the opening up of the magnetic topology. The same feature seems to extend higher up, as can be seen in the online FSI movie created with Jhelioviewer (Müller et al. 2017). Considering that the SR is very close to the EUI solar limb, we believe these images support the link between the CME SR and the filament channel seen in EUVI images (Fig. 3).

\subsection{Metis view of the CME}

The CME first appears in the Metis synoptic set of images starting on 17 January 00:30 UT and remains visible until at least 12:30 UT. Figure 5 summarises the Metis view of the event in the two channels (top two rows), along with their ratio (third row) and the difference image of the two UV images in each dataset (bottom row).

While examining these images, it should be taken into account that features whose projected velocity on the plane of

EUI Data Release 2.0 https://doi .org/10.24414/z2hf-b008 

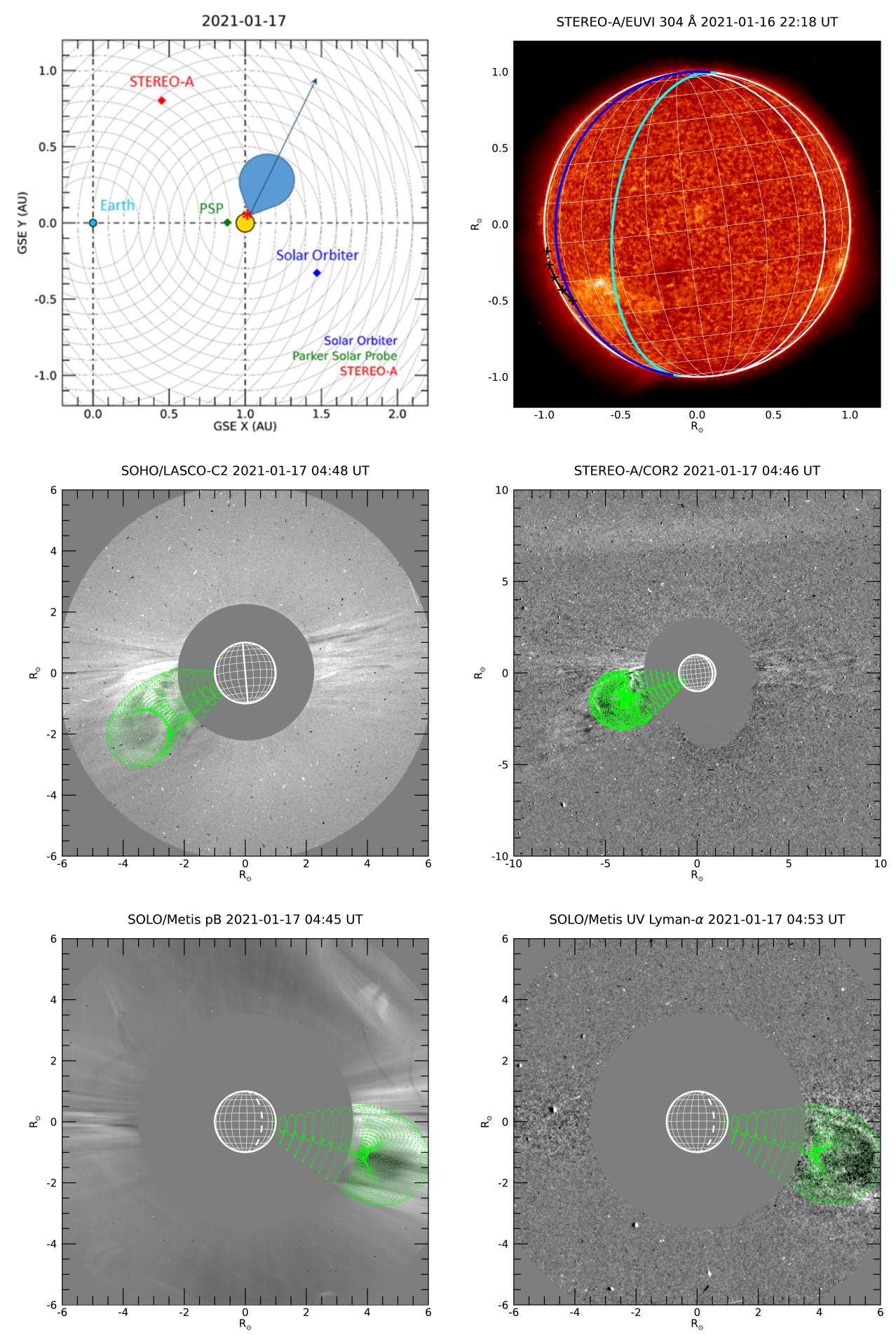

Fig. 2. Top-left panel: location of different spacecraft on 16-17 January 2021 during the CME observed by Metis. The red asterisk on the circle representing the Sun shows the approximate location of the nearby AR (NOAA 12797). The blue arrow indicates the main longitude propagation direction of the CME as determined with GCS fitting. Top-right panel: nearby AR (NOAA 12797) as observed by the STEREO-A/EUVI $30.4 \mathrm{~nm}$ image at 22:18 UT. The asterisk marks the intercept on the solar surface of the vertical axis of the flux rope (see also Fig. 3, which shows the region in more detail), while the connected crosses indicate the projection of the longitudinal axis (the last point is beyond the limb and is connected with a dotted segment). The solid blue and cyan lines represent the position of the solar limb as seen from Solar Orbiter and Earth (and SOHO), respectively. Middle row: GCS reconstruction applied to running-difference images by the SOHO/LASCO-C2 (left) and STEREOA/COR2 (right) instruments. Bottom row: same reconstruction applied to the Solar Orbiter/Metis VL (pB) image (left) and the UV (Ly- $\alpha$ ) image $($ right). The features seen in the NW sector of the Metis/pB image are due to transient spurious reflections occasionally recorded in both channels, possibly due to small debris passing in front of the telescope aperture. In this case, these spurious reflections do not significantly affect the sector of the FOV where the CME is observed. For reference purposes, the Stonyhurst coordinate grid (with $15^{\circ}$ spacing) is overlaid on all the images, along with the central meridian as seen from Earth (solid white line) or the meridian opposite to Earth (dashed white line), whichever of the two is visible on the Sun's disk. 

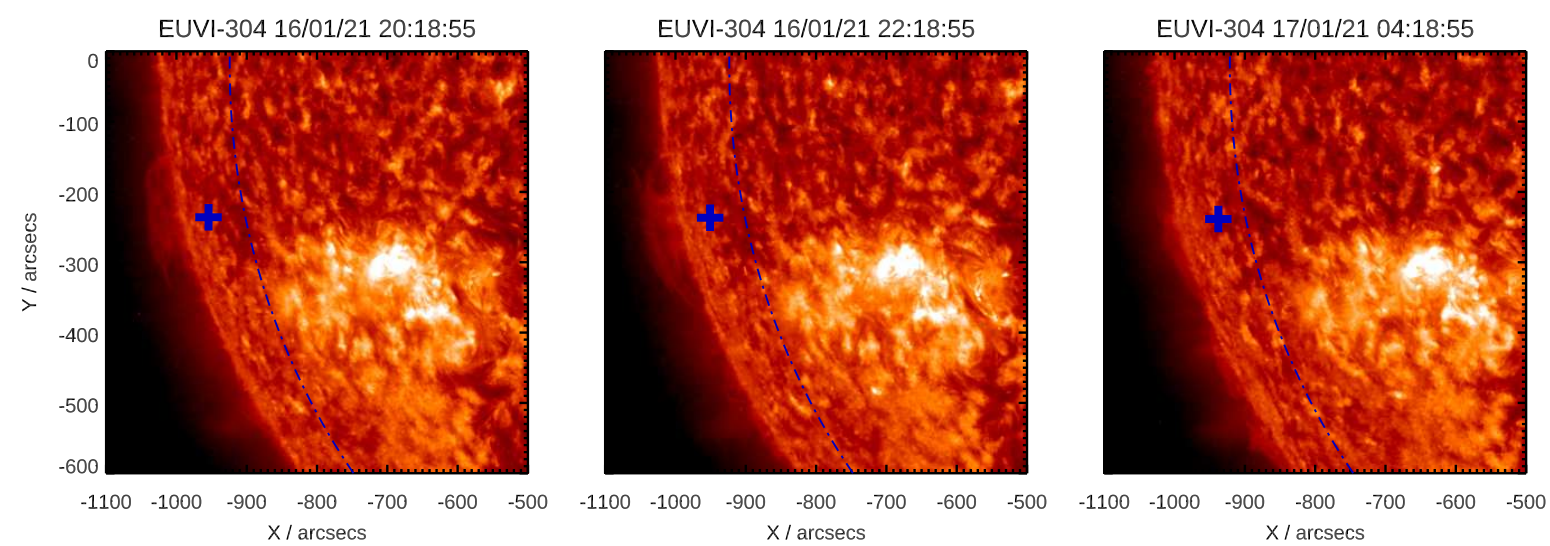

Fig. 3. Area of the likely SR of the 16-17 January 2021 CME as seen by STEREO-A/EUVI in the $30.4 \mathrm{~nm}$ band at three times around the estimated start of the event. The dashed line represents the solar limb as seen by Solar Orbiter/EUI/FSI, while the cross marks the position of the intercept on the solar surface of the vertical axis of the flux rope estimated via GCS reconstruction (see also Fig. 2).
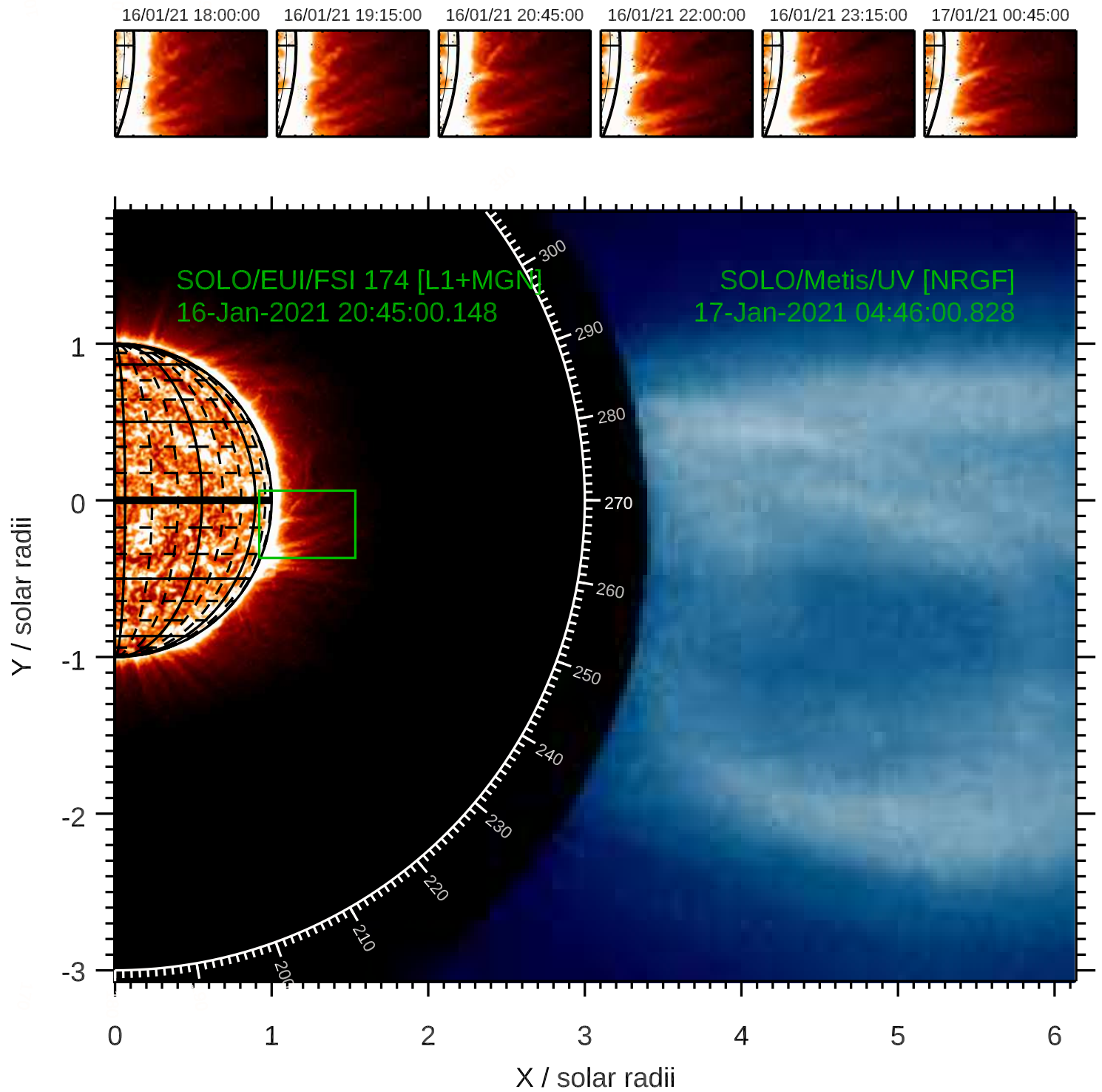

Fig. 4. Metis and EUI view of the 16-17 January 2021 CME, showing both the SR at the start of the event as seen by EUI/FSI $17.4 \mathrm{~nm}$ and the CME at a later time, when it was almost fully within the FOV of the Metis UV channel. The panels in the top row show a time series of closeup EUI/FSI images that correspond to the green box in the larger-scale image below. The EUI/FSI images were processed with the multi-scale Gaussian normalisation (MGN) algorithm (Morgan \& Druckmüller 2014) to enhance small features, while the Metis image was processed with the normalising radial graded filter (NRGF; Morgan et al. 2006) to emphasise structures over the strong gradient of coronal intensities. 


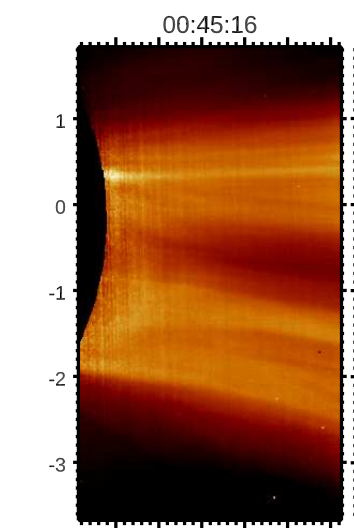

00:53:31
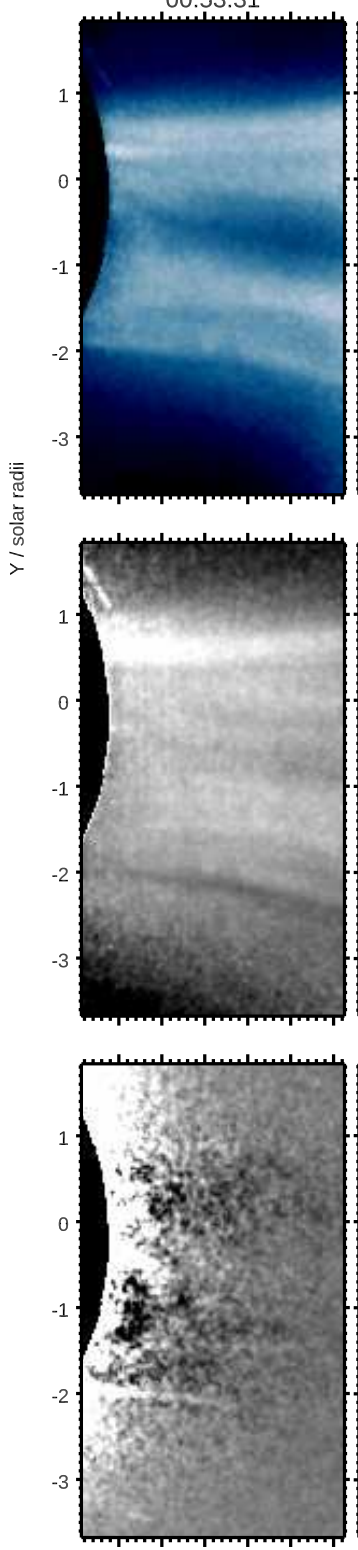

$\begin{array}{llllll}3.5 & 4.0 & 4.5 & 5.0 & 5.5 & 6.0\end{array}$

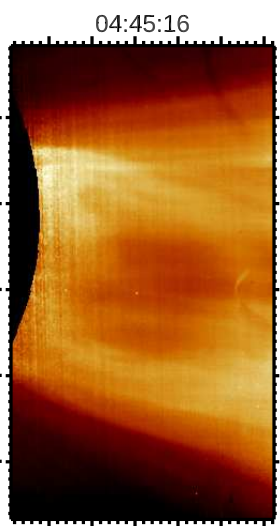

04:53:31
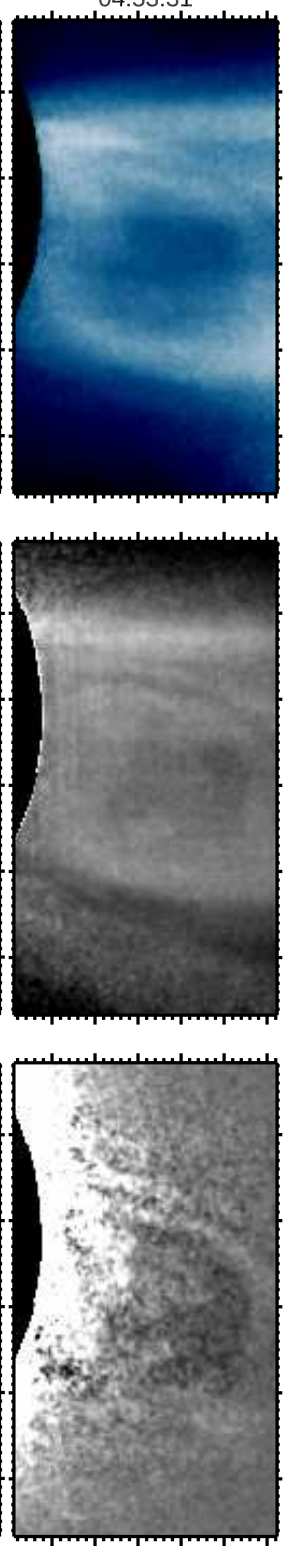

$\begin{array}{llllll}3.5 & 4.0 & 4.5 & 5.0 & 5.5 & 6.0\end{array}$

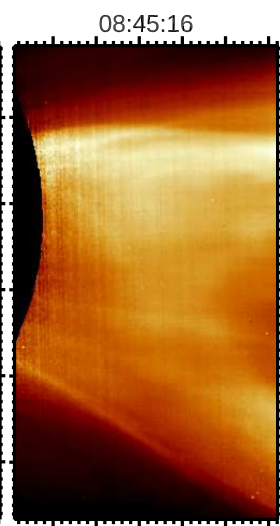

08:53:31
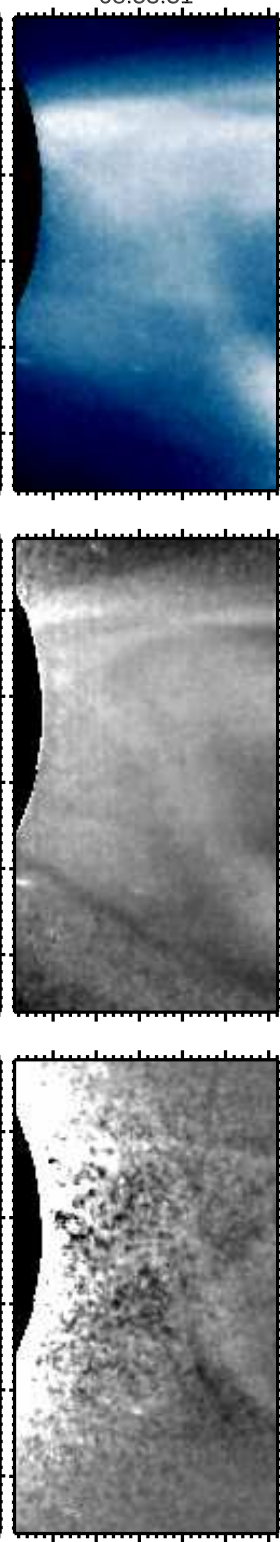

$\begin{array}{llllll}3.5 & 4.0 & 4.5 & 5.0 & 5.5 & 6.0\end{array}$ $\begin{array}{llllll}3.5 & 4.0 & 4.5 & 5.0 & 5.5 & 6.0\end{array}$

$12: 45: 16$

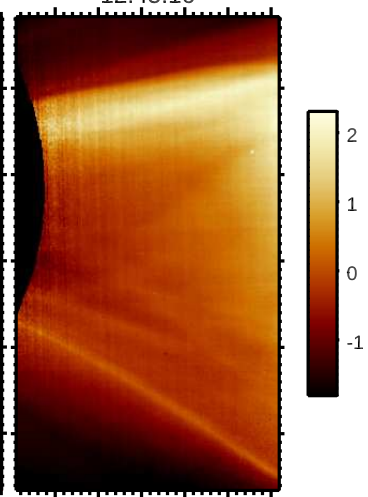

12:53:31
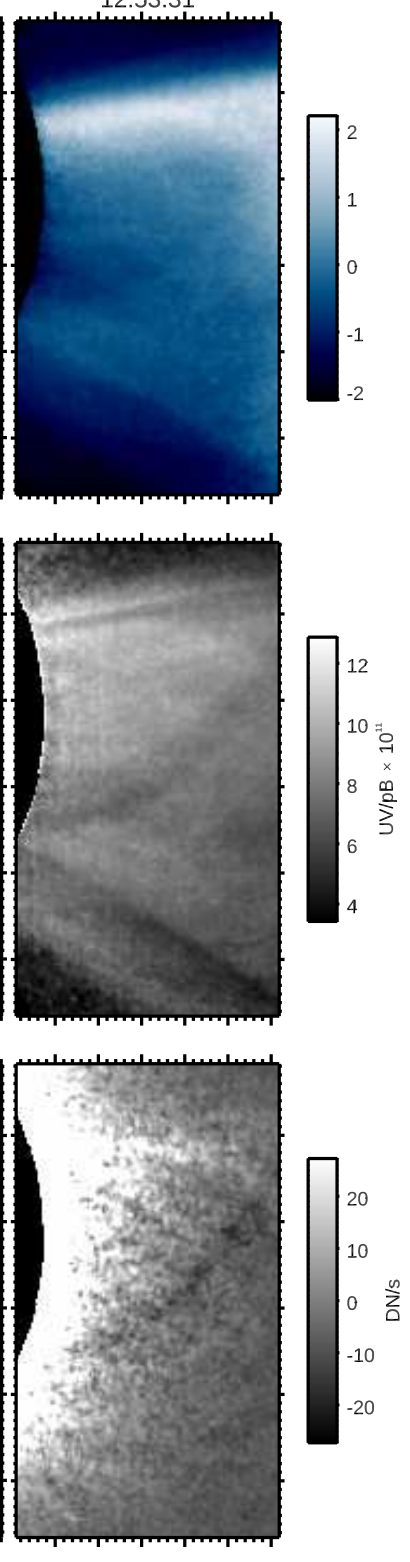

$\mathrm{X} /$ solar radii

Fig. 5. Development of the 16-17 January $2021 \mathrm{CME}$ as observed by the Solar Orbiter/Metis coronagraph. First and second row from the top: VL $\mathrm{pB}$ and UV Ly- $\alpha$ images, respectively, processed via the NRGF algorithm (unit-less numbers as produced by the algorithm). The time labelling each image refers to the centre of the acquisition; in particular, the UV image shown in the second row is the second image in each data acquisition set. Third row: ratio of the UV to $\mathrm{pB}$ images, and last row: difference images obtained from the two pairs of UV images in each data acquisition session. 

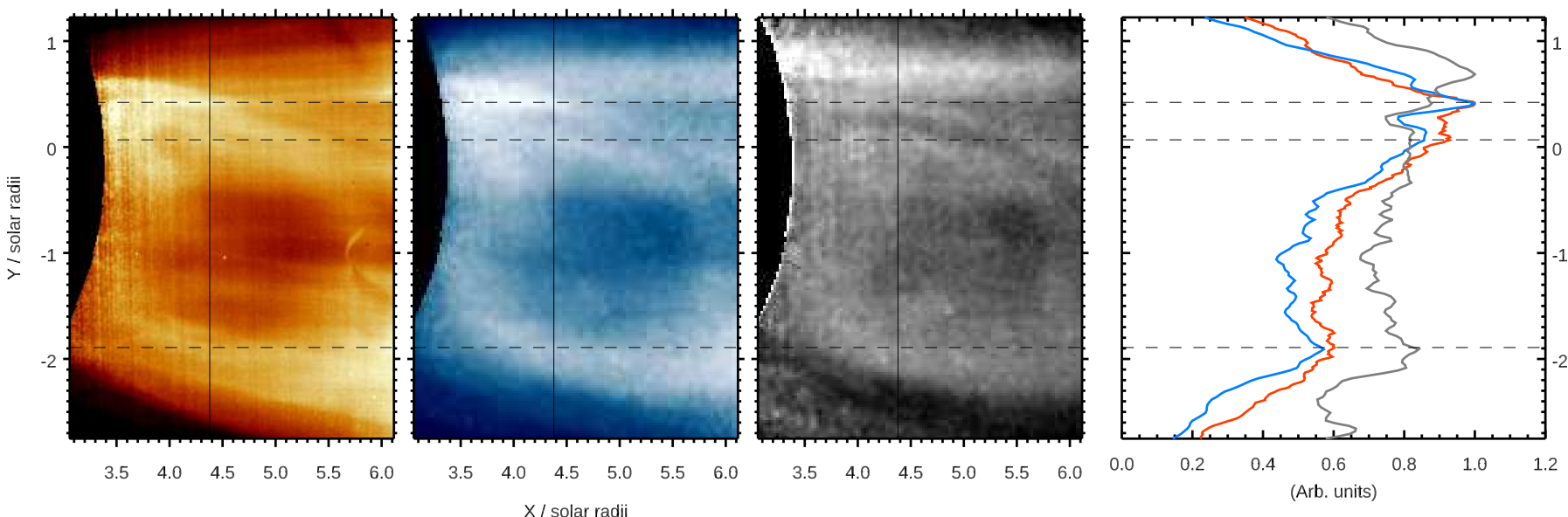

Fig. 6. First three panels: same images shown in the second column of Fig. 5. Right-most panel: count rate profiles along a N-S cut in both Metis channels. The blue and red profiles, each normalised to their peak value, refer to the Ly- $\alpha$ and $\mathrm{pB}$ images, respectively. The grey profile shows the ratio of the two channels along the same cut. The horizontal dashed lines represent fiducials added to aid the identification of features seen in images with those seen in the count rate profiles.

the sky is, for instance, $100 \mathrm{~km} \mathrm{~s}^{-1}$ are seen to move during the $\mathrm{pB}$ and $\mathrm{UV}$ acquisitions by $\sim 20$ and $\sim 5$ pixels, respectively. With this in mind, we note that these images generally show the same features in both channels. While the general appearance of $\mathrm{pB}$ images is more detailed than that of UV images, in part due to the higher resolution of the VL channel (Antonucci et al. 2020), it is striking how some features seen in UV Ly- $\alpha$ images appear more structured than their $\mathrm{pB}$ counterparts. The differences are more clearly seen in the ratio of the two images, shown in the third row of Fig. 5, where the $\mathrm{pB}$ images were degraded in resolution to match the resolution of the UV images. For instance, the separation between the leading fronts in the 04:53 images seems more evident in Ly- $\alpha$ than in $\mathrm{pB}$ images. The CME cavity, on the other hand, has a similar depth in both channels. To better illustrate both points, we show in Fig. 6 the profiles in a N-S cut at $\sim 4.4 R_{\odot}$ in the 04:45 (pB) and 04:53 (Ly- $\alpha$ ) images. The image profiles in the cavity are remarkably similar. However, the gap between the two $\mathrm{N}$ fronts, for instance, is evident in the Ly$\alpha$ profiles but much less so in the $\mathrm{pB}$ profile. On the other hand, the $\mathrm{S}$ front seems narrower in Ly- $\alpha$ than in $\mathrm{pB}$. Finally, we do not find evidence of a bright core, which would have been a possible signature of the eruptive filament (but see also Howard et al. 2017).

We note that the last two images of the sequence seem to show motions compatible with some untwisting features. Similar features were also reported by Antonucci et al. (1997) and Ciaravella et al. (2000) using UVCS spectroscopic data.

With the caveats mentioned in Appendix A, we compared the pairs of UV Ly- $\alpha$ images taken in each acquisition session, in particular those taken between 04:30-04:45 UT and 04:4605:00 UT on 17 January (last row of Fig. 5), a time interval when the CME was almost entirely within the Metis FOV. We were thus able to track the location of multiple nested loop-like features visible in the UV images. These features are shown in Fig. 7; from an elliptical fitting to the location of the inner front, we derived an expansion velocity of the order of $\sim 140 \mathrm{~km} \mathrm{~s}^{-1}$, while the centre of the CME flux rope (assumed to be tracked by the centre of the fitting ellipse) expands at the much lower velocity of $70 \mathrm{~km} \mathrm{~s}^{-1}$. In comparison, the wind speed in the corona through which the CME propagates is likely to be similar or higher: Telloni et al. (2021) estimate from the same dataset,

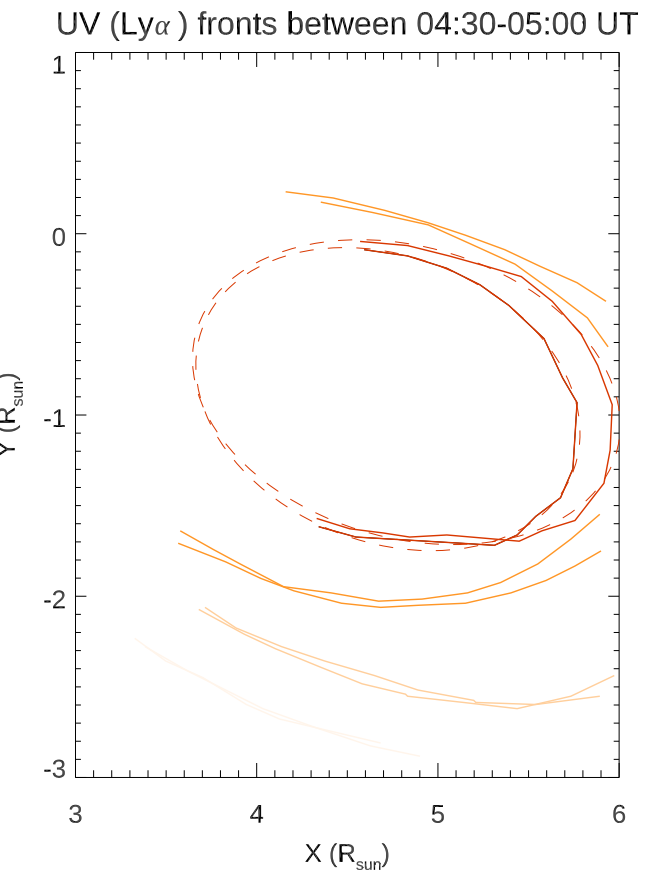

Fig. 7. Tracked locations of multiple UV fronts as observed by Metis on 16-17 January 2021 between 04:30 and 05:00 UT. The inner front locations measured at two different times (solid red line) have been fitted with an ellipse (dashed red line) to measure the CME centre and front speeds (see text). The orange and yellow lines identify the locations of two other fronts visible in the images.

using a Doppler dimming technique, the coronal wind speed in the streamer over the east limb to be $140 \mathrm{~km} \mathrm{~s}^{-1}$ at $4 R_{\odot}$, and $180 \mathrm{~km} \mathrm{~s}^{-1}$ at $6.5 R_{\odot}$. Likewise, Romoli et al. (2021) report Metis measurements of wind speeds higher than $150 \mathrm{~km} \mathrm{~s}^{-1}$ in a corona that displays a similar configuration. Clearly, these data demonstrate that Metis is capable of providing information on radial velocities for both the background corona and for the $\mathrm{CME}$, thus providing valuable information for the study of the mechanisms of CME propagation (see e.g., the review by Chen 2011).

We note that the overall similarity between the $\mathrm{pB}$ and UV images is in apparent contradiction with the results by 
Bemporad et al. (2018), who predicted, for instance, that the CME front should appear darker in Ly- $\alpha$ than the coronal background environment. We also note that while this prediction was valid for a simulated fast CME (radial velocities well in excess of $300 \mathrm{~km} \mathrm{~s}^{-1}$ ), for which the Doppler dimming effect strongly reduces the Ly- $\alpha$ CME emission from the front, the expansion speed of the event reported here is much lower. In more general terms, the Doppler dimming of coronal lines depends on various factors (Noci et al. 1987), notably density and incident (chromospheric) radiation. For a rough comparison between the Bemporad et al. (2018) simulations and these observations, we refer to Fig. 1 of Dolei et al. (2018): The reduction factor of the Ly- $\alpha$ line with respect to the stationary case is $\sim 0.75$ for a radial velocity of $\sim 150 \mathrm{~km} \mathrm{~s}^{-1}$ and $<0.1$ for radial velocities $>300 \mathrm{~km} \mathrm{~s}^{-1}$. The case modelled by Bemporad et al. (2018) falls into the latter category (see also their Fig. 8), while the CME described here is better represented by the former case. This is consistent with the remarkable similarities of the UV and $\mathrm{pB}$ profiles shown in Fig. 6, which we think are compatible with Ly- $\alpha$ emission mainly following the electron density distributions in the corona with a similar dependence as the $\mathrm{pB}$ emission.

It should also be taken into account that both nonequilibrium ionisation effects (Pagano et al. 2020), which produce a higher fraction of neutral $\mathrm{H}$ atoms in the $\mathrm{CME}$ front with respect to the equilibrium case, and lower temperatures could increase the UV emission in the Ly- $\alpha$ line (e.g., Vial \& Chane-Yook 2016).

\section{Conclusions}

In this Letter we describe the first CME observed by the Metis coronagraph on board Solar Orbiter. The event, observed on 1617 January 2021, was relatively faint and slow, and the observations were taken during a low-cadence synoptic run (one dataset every 4 hours). Nonetheless, the data quality, the multiwavelength view of the event, and the simultaneous observations with the EUI/FSI imager provided an excellent preview of the capabilities of Metis for observing CMEs.

In particular, we were able to identify the CME's most likely SR in a filament channel next to AR NOAA 12797. The observed event can be classified as a 'stealth' CME, that is, a CME without low coronal signatures (Robbrecht et al. 2009), although some faint features are seen in the EUI images.

For the first time, we show simultaneous images of a CME both in VL (pB) and in Ly- $\alpha$. Overall, Metis images in both channels show essentially the same features, although some significant differences could be identified at small scales. We estimated the expansion velocity of the CME fronts seen in Ly$\alpha$ to be of the order of $\sim 140 \mathrm{~km} \mathrm{~s}^{-1}$. With these velocities it is unlikely that the Doppler dimming effect on Ly- $\alpha$ emission could explain the observed differences. Nevertheless, measurement of velocities as done in this case will be important for constraining the amount of Doppler dimming of the Ly- $\alpha$ emission from CME plasma needed for a physical interpretation and modelling of the observed emission. Moreover, these velocities, along with estimates of the radial wind speed in the corona obtained from Doppler dimming techniques from the same data, can provide a unique set of measurements for studying the physical mechanisms of CME acceleration and propagation.

Finally, by combining EUI/FSI (up to $1.9^{\circ}$ from Sun centre) and Metis data (from $1.6^{\circ}$ ), the possibility of providing further constraints on the temperature of CME features and of the ambient corona emerges.

Acknowledgements. Solar Orbiter is a space mission of international collaboration between ESA and NASA, operated by ESA. The Metis programme is supported by the Italian Space Agency (ASI) under the contracts to the co-financing National Institute of Astrophysics (INAF): Accordi ASI-INAF N. I-043-10-0 and Addendum N. I-013-12-0/1, Accordo ASI-INAF N.2018-30-HH.0 and under the contracts to the industrial partners OHB Italia SpA, Thales Alenia Space Italia SpA and ALTEC: ASI-TASI N. I-037-11-0 and ASI-ATI N. 2013-057-I.0. Metis was built with hardware contributions from Germany (Bundesministerium für Wirtschaft und Energie (BMWi) through the Deutsches Zentrum für Luft- und Raumfahrt e.V. (DLR)), from the Academy of Sciences of the Czech Republic (Czech PRODEX) and from ESA. The EUI instrument was built by CSL, IAS, MPS, MSSL/UCL, PMOD/WRC, ROB, LCF/IO with funding from the Belgian Federal Science Policy Office (BELSPO/PRODEX PEA 4000134474, PEA 4000134088); the Centre National d'Etudes Spatiales (CNES); the UK Space Agency (UKSA); the Bundesministerium für Wirtschaft und Energie (BMWi) through the Deutsches Zentrum für Luft- und Raumfahrt (DLR); and the Swiss Space Office (SSO). The SOHO/LASCO data used here are produced by a consortium of the Naval Research Laboratory (USA), Max-Planck-Institut für Aeronomie (Germany), Laboratoire d'Astronomie (France), and the University of Birmingham (UK). (MPI for Aeronomy (MPAe) has changed its name to the Max-Planck-Institut for Sonnensystemforschung (MPS), and the Laboratoire d'Astronomie (LAS) has changed its name to the Laboratoire d'Astrophysique Marseille (LAM).) SOHO is a project of international cooperation between ESA and NASA. The SECCHI data are produced by an international consortium of the NRL, LMSAL, and NASA GSFC (USA), RAL and U. Bham (UK), MPS (Germany), CSL (Belgium), IOTA, and IAS (France). L.S. was supported by a grant from the NASA Heliophysics Technology and Instrument Development for Science Program, NNH15ZDA001NHTIDS, and also by the basic Research Funds of the Office of Naval Research.

\section{References}

Antonucci, E., Kohl, J. L., Noci, G., et al. 1997, ApJ, 490, L183 Antonucci, E., Romoli, M., Andretta, V., et al. 2020, A\&A, 642, A10 Auchère, F., Andretta, V., Antonucci, E., et al. 2020, A\&A, 642, A6 Bemporad, A., Pagano, P., \& Giordano, S. 2018, A\&A, 619, A25 Brueckner, G. E., Howard, R. A., Koomen, M. J., et al. 1995, Sol. Phys., 162, 357

Chen, P. F. 2011, Liv. Rev. Sol. Phys., 8, 1

Ciaravella, A., Raymond, J. C., Thompson, B. J., et al. 2000, ApJ, 529, 575

Cremades, H., \& Bothmer, V. 2004, A\&A, 422, 307

Cremades, H., Bothmer, V., \& Tripathi, D. 2006, Adv. Space Res., 38, 461

Dolei, S., Susino, R., Sasso, C., et al. 2018, A\&A, 612, A84

Fineschi, S., Naletto, G., Romoli, M., et al. 2020, Exp. Astron., 49, 239

Fox, N. J., Velli, M. C., Bale, S. D., et al. 2016, Space Sci. Rev., 204, 7

Giordano, S., Ciaravella, A., Raymond, J. C., Ko, Y. K., \& Suleiman, R. 2013, J. Geophys. Res. (Space Phys.), 118, 967

Gopalswamy, N., Yashiro, S., Michalek, G., et al. 2009, Earth Moon Planets, 104,295

Howard, R. A., Moses, J. D., Vourlidas, A., et al. 2008, Space Sci. Rev., 136, 67 Howard, T. A., DeForest, C. E., Schneck, U. G., \& Alden, C. R. 2017, ApJ, 834, 86

Kohl, J. L., Esser, R., Gardner, L. D., et al. 1995, Sol. Phys., 162, 313

Kohl, J. L., Noci, G., Cranmer, S. R., \& Raymond, J. C. 2006, A\&ARv, 13, 31 Lamy, P. L., Floyd, O., Boclet, B., et al. 2019, Space Sci. Rev., 215, 39

Michalek, G., Gopalswamy, N., Lara, A., \& Yashiro, S. 2006, Space Weather, 4, $\mathrm{S} 10003$

Morgan, H., \& Druckmüller, M. 2014, Sol. Phys., 289, 2945

Morgan, H., Habbal, S. R., \& Woo, R. 2006, Sol. Phys., 236, 263

Müller, D., Nicula, B., Felix, S., et al. 2017, A\&A, 606, A10

Müller, D., St. Cyr, O. C., Zouganelis, I., et al. 2020, A\&A, 642, A1

Nicula, B., Berghmans, D., \& Hochedez, J.-F. 2005, Sol. Phys., 228, 253

Noci, G., Kohl, J. L., \& Withbroe, G. L. 1987, ApJ, 315, 706

Pagano, P., Bemporad, A., \& Mackay, D. H. 2020, A\&A, 637, A49

Robbrecht, E., \& Berghmans, D. 2004, A\&A, 425, 1097

Robbrecht, E., Patsourakos, S., \& Vourlidas, A. 2009, ApJ, 701, 283

Rochus, P., Auchère, F., Berghmans, D., et al. 2020, A\&A, 642, A8

Romoli, M., Antonucci, E., Andretta, V., et al. 2021, A\&A, 656, A32 (SO Cruise Phase SI)

Schwenn, R., dal Lago, A., Huttunen, E., \& Gonzalez, W. D. 2005, Ann. Geophys., 23, 1033

Telloni, D., Andretta, V., Antonucci, E., et al. 2021, ApJ, 920, L14

Thernisien, A. F. R. 2011, ApJ, 194, 33 
Thernisien, A. F. R., Howard, R. A., \& Vourlidas, A. 2006, ApJ, 652, 763

Vial, J.-C., \& Chane-Yook, M. 2016, Sol. Phys., 291, 3549

Vourlidas, A., Subramanian, P., Dere, K. P., \& Howard, R. A. 2000, ApJ, 534, 456

Vourlidas, A., Howard, R. A., Esfandiari, E., et al. 2010, ApJ, 722, 1522

Webb, D. F., \& Howard, T. A. 2012, Liv. Rev. Sol. Phys., 9, 3

Webb, D. F., Cliver, E. W., Crooker, N. U., Cry, O. C. S., \& Thompson, B. J. 2000, J. Geophys. Res., 105, 7491

Xie, H., Ofman, L., \& Lawrence, G. 2004, J. Geophys. Res. (Space Phys.), 109, A03109

Yashiro, S., Gopalswamy, N., Michalek, G., et al. 2004, J. Geophys. Res. (Space Phys.), 109, A07105

Zhang, J., Dere, K. P., Howard, R. A., \& Vourlidas, A. 2004, ApJ, 604, 420

1 INAF - Osservatorio Astronomico di Capodimonte, Salita Moiariello 16, 80131 Naples, Italy e-mail: vincenzo. andretta@inaf.it

2 INAF - Osservatorio Astrofisico di Torino, Turin, Italy

3 Max-Planck-Institut für Sonnensystemforschung, Göttingen, Germany

4 INAF - Osservatorio Astronomico di Trieste, Trieste, Italy

5 Royal Observatory of Belgium, Brussels, Belgium

6 Institute of Geodynamics of the Romanian Academy, Bucharest, Romania

7 Università di Padova - Dip. Fisica e Astronomia “Galileo Galilei”, Padua, Italy

8 CNR - Istituto di Fotonica e Nanotecnologie, Padua, Italy

9 INAF - Associate Scientist, Italy

10 Università di Firenze - Dip. Fisica e Astronomia, Florence, Italy

11 INAF - Osservatorio Astrofisico di Catania, Catania, Italy

12 KU Leuven, Leuven, Belgium
13 INAF - Istituto di Astrofisica Spaziale e Fisica Cosmica, Milan, Italy

14 Université Paris-Saclay, CNRS, Institut d'Astrophysique Spatiale, Orsay, France

15 University of Wrocław - Astronomical Institute,, Poland

16 Università di Catania - Dip. Fisica e Astronomia "Ettore Majorana", Catania, Italy

17 Università di Padova - CISAS, Padua, Italy

18 Catholic University of America, Washington, DC, USA

19 Università di Urbino "Carlo Bo" - DiSPeA, Urbino, Italy

20 INFN - Sez. Firenze, Florence, Italy

21 Astronomical Institute of the Czech Academy of Sciences, Ondřejov, Czech Republic

22 Università di Torino, Turin, Italy

23 Politecnico di Torino - DET, Turin, Italy

24 NASA HQ, Washington, DC, USA

25 CNR - IEIIT, Padua, Italy

26 Agenzia Spaziale Italiana, Roma, Italy

27 INAF - Istituto di Astrofisica e Planetologia Spaziali di Roma, Rome, Italy

28 INAF - Osservatorio Astronomico di Palermo, Palermo, Italy

29 Laboratoire Atmosphères, Milieux et Observations Spatiales, Guyancourt, France

30 Università di Pavia, Pavia, Italy

31 Università di Padova, Padua, Italy

32 Università della Calabria - Dip, Fisica, Italy

33 Università di Palermo - Dip. Fisica e Chimica "Emilio Segrè", Palermo, Italy

34 Naval Research Laboratory, Washington, DC, USA

35 University of Athens, Dep. Physics, Athens, Greece

36 University of California, Los Angeles, California, USA 


\section{Appendix A: Updates to the Metis calibration}

The Metis on-ground and in-flight calibration as determined at the end of the instrument commissioning are summarised in Antonucci et al. (2020) and Romoli et al. (2021), respectively. Here we provide some updates that are relevant for the analysis of the 16-17 January event.

\section{A.1. Dealing with transient effects of the UV detector}

The detector of the UV Ly- $\alpha$ channel is subject, in its current mode of operations, to a transient effect that reduces the response of the images acquired in the first $\sim 90 \mathrm{~s}$ after the command initiating the acquisition sequence. This effect is known and has already been measured during on-ground test campaigns at the instrument level, although the very short time available for such campaigns did not allow us to fully assess the problem. However, by analysing in-flight UV Ly- $\alpha$ channel data taken during Commissioning and early Cruise operations, we have been able to determine the magnitude of that effect. The effect is non-linear with count rates, thus making the radiometric calibration of those images problematic. In the particular case of the synoptic observations analysed in this work, the effect is modest, of the order of $5 \%$ over the FOV. For most of the analysis presented in this work, it was therefore possible to utilise both UV images in each acquisition sequence.

The same transient effect also makes the UV dark frames acquired just before the synoptic run in principle not directly applicable to this dataset, as the acquisition parameters were not exactly the same as for the synoptic set. We therefore utilised the dark frames acquired at a later date (20 April 2021). We, however, verified that the use of January 2021 dark frames corrected with an empirical algorithm produces very similar results: The difference is less than seven digital numbers (DNs) for $70 \%$ of the pixels. The empirical correction algorithm, currently not the baseline in the processing pipeline, takes into account the different effect of the transient on acquisitions averaged over a different number of frames and was tested on various sets of dark frames acquired close in time but with different parameters.

The effect of the transient is still not negligible in some regions of the detector, especially near the edge of the occulter, where large gradients of count rates occur; nevertheless, the effect in most areas of the detector is less important, allowing us, for example, to examine the morphology of the CME and of its velocity field along the plane of the sky. In addition, and for the same reason, we did not apply radiometric calibration factors, leaving UV data in count rate units $\left(\mathrm{DN} \mathrm{s}^{-1}\right)$.

We are currently testing an update of the on-board operating software on the ground reference model of the detection subsystem, which should mitigate, and possibly eliminate, the effect of the transient on the Metis UV Ly- $\alpha$ data.

\section{A.2. The UV vignetting function}

In order to correctly account for variations in the relative brightness of images in the two channels, VL and UV, we applied the first results of an ongoing investigation of the relative efficiency of the two channels. The adopted method exploits the fact that

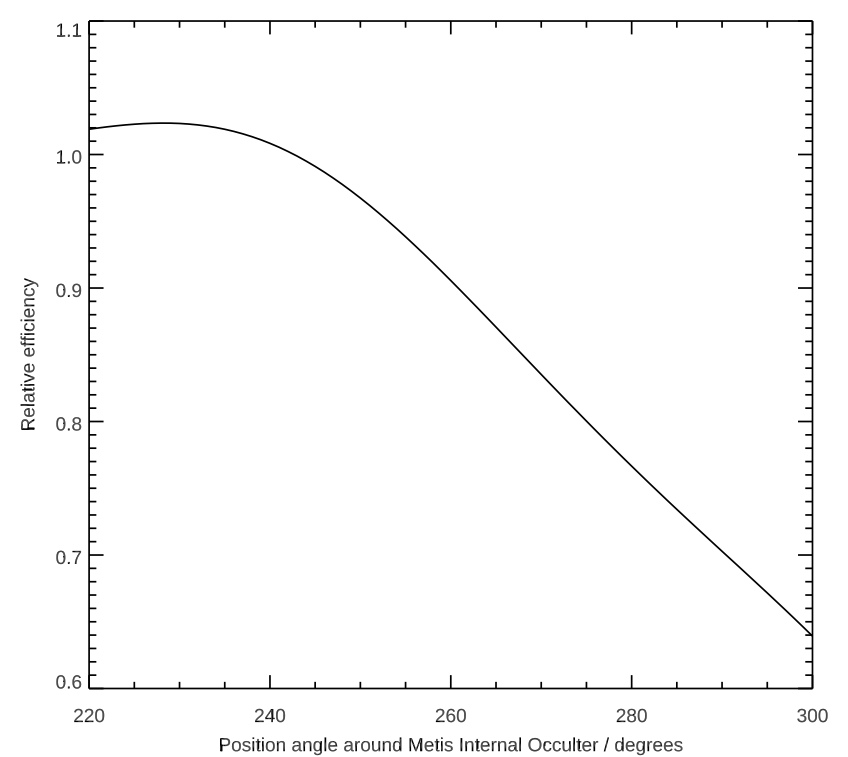

Fig. A.1. Relative efficiency of the Metis UV channel with respect to the VL channel in the sector where the CME was observed.

the Metis heat shield (HS) door is not light-tight: When closed, a significant amount of radiation is back-reflected into the instrument in both channels. Assuming that the ratio of brightness patterns of the door seen at the focal planes of the two channels is approximately constant, the ratio of the UV and VL images of the closed HS door provides a map of the variability of the relative efficiency of the two channels over the FOV or, equivalently, a map of the ratio of the respective vignetting functions.

These UV-to-VL ratio images of the closed HS door show little evidence of systematic gradients in the radial direction; the main variation occurs in the azimuthal direction, taking as reference point the centre of the internal occulter shadow. We therefore averaged those images in the radial direction, smoothing the resulting function of the azimuth in order to remove any residual small-scale features of the ratio of the brightness patterns. The resulting function, valid in the angular sector of the detector where the CME analysed in this work is imaged, is shown in Fig. A.1. The correction factor shown in that figure was then applied to the standard UV vignetting function.

This approach in correcting the UV vignetting function was validated through the analysis of the transits in the instrument FOV in June 2020 of the calibration stars $\alpha$ and $\rho$ Leo across the equatorial region of the Metis FOV. The same analysis has shown that the large-scale variations seen from the HS door back-illumination are primarily due to the UV channel.

Work is in progress to extend this analysis to other areas of the detector, in particular to the north polar regions, by analysing more images of the closed HS door and more stellar transits, as well as data taken during spacecraft roll manoeuvres. We also remark that the correction to the UV vignetting function obtained with this method is consistent with the previous correction adopted in Romoli et al. (2021), also applicable to equatorial areas only, which was based solely on stellar radiometric measurements. 